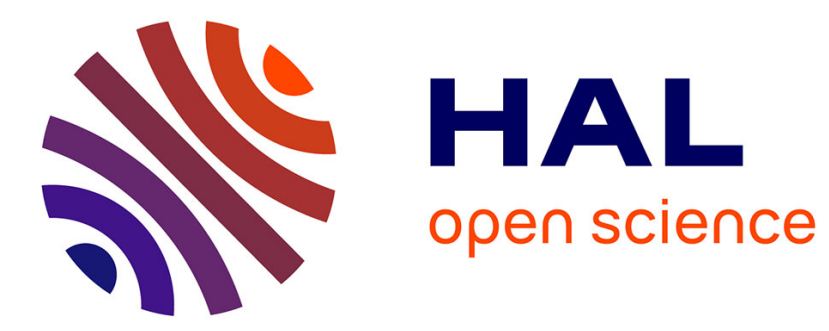

\title{
A Functional CLT for Fields of Commuting Transformations Via Martingale Approximation
}

Christophe Cuny, Jerome Dedecker, Dalibor Volny

\section{To cite this version:}

Christophe Cuny, Jerome Dedecker, Dalibor Volny. A Functional CLT for Fields of Commuting Transformations Via Martingale Approximation. Zapiski Nauchnyh Seminarov POMI, 2016, 441 (5), pp.239-262. 10.1007/s10958-016-3145-y . hal-01261297

\section{HAL Id: hal-01261297 \\ https://hal.science/hal-01261297}

Submitted on 25 Jan 2016

HAL is a multi-disciplinary open access archive for the deposit and dissemination of scientific research documents, whether they are published or not. The documents may come from teaching and research institutions in France or abroad, or from public or private research centers.
L'archive ouverte pluridisciplinaire HAL, est destinée au dépôt et à la diffusion de documents scientifiques de niveau recherche, publiés ou non, émanant des établissements d'enseignement et de recherche français ou étrangers, des laboratoires publics ou privés. 


\title{
A FUNCTIONAL CLT FOR FIELDS OF COMMUTING TRANSFORMATIONS VIA MARTINGALE APPROXIMATION
}

\author{
CHRISTOPHE CUNY, JÉRÔME DEDECKER, AND DALIBOR VOLNÝ \\ Dedicated to the memory of Mikhail (Misha) Gordin
}

\begin{abstract}
We consider a field $f \circ T_{1}^{i_{1}} \circ \ldots \circ T_{d}^{i_{d}}$, where $T_{1}, \ldots, T_{d}$ are completely commuting transformations in the sense of Gordin. If one of these transformations is ergodic, we give sufficient conditions in the spirit of Hannan under which the partial sum process indexed by quadrants converges in distribution to a brownian sheet. The proof combines a martingale approximation approach with a recent CLT for martingale random fields due to Volný. We apply our results to completely commuting endomorphisms of the $m$-torus. In that case, the conditions can be expressed in terms of the $L^{2}$-modulus of continuity of $f$.
\end{abstract}

\section{INTRODUCTION}

Let $(\Omega, \mathcal{A}, \mu)$ be a probability space and $T$ a (non-invertible) measure preserving map. Let $U$ be the associated Koopman operator $\left(U f=f \circ T\right.$ for every $f \in L^{1}(\Omega, \mathcal{A}, \mu)$ ) and $U^{*}$ be the associated Perron-Frobenius operator. In 1978, Gordin and Lifšic [10] (see also [8]) observed that if $f=\left(I-U^{*}\right) g$ for some $g \in L^{2}(\mu)$ (i.e. $f$ is a coboundary for $\left.U^{*}\right)$, then one has a decomposition $f=\left(g-U U^{*} g\right)+(U-I) U^{*} g$ into the sum of a reverse martingale difference plus a coboundary (for $U$ that time). This allows to prove the central limit theorem (CLT) and the weak invariance principle (WIP) for $f$ from the corresponding results for stationary reverse martingale differences.

This fruitful approach presents a part of the martingale-approximation method, known also as Gordin's method and started with the seminal paper [8] from 1969. It has been further developped in many papers (to go beyond the coboundaries for $\left.U^{*}\right)$. Let us mention the following references where optimal or sharp results have been obtained concerning the CLT as well as other limit theorems: Hannan [13, 14], Heyde [15], Maxwell and Woodroofe [17], Peligrad and Utev [18], Gordin and Peligrad [11], Cuny [4].

Consider now a family of commuting measure preserving transformations $T_{1}, \ldots, T_{d}$. In 2009, Gordin [9] proved a decomposition analogous to the above one (see Section 4 ), when the transformations are completely commuting (see the next section for the definition) and $f=\left(I-U_{1}^{*}\right) \cdots\left(I-U_{d}^{*}\right) g$. However, probably by lack of a CLT for

1991 Mathematics Subject Classification. Primary: 60J05, 60J55, 47A35, 28D05, 37A05; Secondary: $47 \mathrm{~B} 38$.

Key words and phrases. random fields, reverse martingales, endomorphisms of the torus. 
"multi-dimensional" reverse martingale differences, he did not derive any CLT from that decomposition.

Very recently, the third author [19] proved such a CLT. Actually, he worked in the setting of martingale differences but, as shown in Section 3, its proof applies equally in the reverse martingale case and yields also the weak invariance principle. In this paper, we provide a suitable reverse-martingale approximation under a condition in the spirit of Hannan, from which the CLT and the WIP follow. Note that the WIP under Hannan's condition has been recently obtained by Volný and Wang [20] in the case where the random field can be expressed as a function of an iid random field. In the one-dimensional setting this condition is known to be sharp (see for instance Dedecker [6]). The results of Volný and Wang can, using [20], be extended to random fields which are not Bernoulli.

We apply these results to prove a CLT and a WIP in the case where the transformations are commuting dilating endomorphisms of the $m$-dimensional torus. Note that, for such commuting endomorphisms (not necessarily dilating), the CLT has been obtained recently by mean of completely different technics under slightly stronger conditions, see Section 5 for a deeper discussion.

\section{Setting of the PAPER}

Let us describe our setting.

Let $(\Omega, \mathcal{A}, \mu)$ be a probability space. Consider a family $\left\{T_{1}, \ldots, T_{d}\right\}$ of measure preserving transformations on $\Omega$.

Denote by $U_{1}, \ldots, U_{d}$ the corresponding Koopman operators and by $U_{1}^{*}, \ldots, U_{d}^{*}$ the associated adjoint operators, also known in that context as the Perron-Frobenius operators. Recall that those operators are characterized as follows

$$
\begin{aligned}
U_{i} f & =f \circ T_{i} \\
\int_{\Omega} U_{i} f g d \mu & =\int_{\Omega} f U_{i}^{*} g d \mu,
\end{aligned}
$$

for every positive measurable functions $f, g$ and every $i \in\{1, \ldots, d\}$.

Definition 1. We say that the family $\left\{T_{1}, \ldots, T_{d}\right\}$ (or the family $\left\{U_{1}, \ldots, U_{d}\right\}$ ) is completely commuting if it is commuting and if moreover

$$
U_{i} U_{j}^{*}=U_{j}^{*} U_{i} \quad \text { for any } i, j \in\{1, \ldots, d\}, i \neq j .
$$

Notice that (as already observed by Gordin) this definition is slightly abusive since that property depends on $\mu$. Since $\mu$ will be fixed in the sequel, we will not worry about that fact.

We consider now the natural filtrations associated with our transformations. For every $i \in\{1, \ldots, d\}$ and every $n \in \mathbb{N}$, denote $\mathcal{F}_{n}^{(i)}:=T_{i}^{-n}(\mathcal{A})$.

Then, it is well-known (and not hard to prove) that, for every $i \in\{1, \ldots, d\}$ and every $n \in \mathbb{N}$, we have 


$$
\mathbb{E}\left(f \mid \mathcal{F}_{n}^{(i)}\right)=U_{i}^{n}\left(U_{i}^{*}\right)^{n} f \quad \text { for any } f \in L^{1}(\Omega, \mathcal{A}, \mu) .
$$

On the other hand, since the $U_{i}$ 's are clearly isometries (of any $L^{p}, 1 \leq p \leq \infty$ ), we also have for every $i \in\{1, \ldots, d\}$

$$
U_{i}^{*} U_{i} f=f \quad \text { for any } f \in L^{1}(\Omega, \mathcal{A}, \mu) .
$$

The relevance of the property of complete commutation lies in the fact that for every $i, j \in\{1, \ldots, d\}$ with $i \neq j$, and every $n \in \mathbb{N}$, the operator $U_{i}$ and the operator of conditional expectation with respect to $\mathcal{F}_{n}^{(j)}$ are commuting.

\section{AN INVARIANCE PRINCIPLE FOR STATIONARY $d$-FIELDS OF REVERSE MARTINGALE DIFFERENCES}

In all of this section we suppose given a completely commuting family $\left\{T_{1}, \ldots, T_{d}\right\}$ of transformations on the probability space $(\Omega, \mathcal{A}, \mu)$ and we make use of the previous notations.

We shall use the notation $\underline{n}$ to specify that $\underline{n}$ is a vector. Then, if $\underline{n}=\left(n_{1}, \ldots, n_{d}\right) \in$ $\mathbb{N}^{d}$ (with $\mathbb{N}=\{0,1 \ldots\}$, and later $\mathbb{N}^{*}=\{1,2 \ldots\}$ ), we shall use the notation

$$
U^{\underline{n}} f=U_{1}^{n_{1}} \cdots U_{d}^{n_{d}} f \quad \text { for any } f \in L^{1}(\Omega, \mathcal{A}, \mu) .
$$

Definition 2. We shall say that $\left(f_{\underline{n}}\right)_{\underline{n} \in \mathbb{N}^{d}}$ is a commuting stationary d-field of reverse martingale differences if there exists $f \in L^{1}(\Omega, \mathcal{A}, \mu)$ with $\mathbb{E}\left(f \mid \mathcal{F}_{1}^{(i)}\right)=0$ for every $i \in\{1, \ldots, d\}$ such that $f_{\underline{n}}=U^{\underline{n}} f$.

Let $\left\{e_{1}, \ldots, e_{d}\right\}$ be the canonical basis in $\mathbb{R}^{d}$. If $\left(f_{\underline{n}}\right)_{\underline{n} \in \mathbb{N}^{d}}$ is a commuting stationary $d$-field of reverse martingale differences, then, for every $i \in\{1, \ldots, d\}$, we have (with $f$ as in the definition), $U_{i}^{*} f=0$ and

$$
\mathbb{E}\left(f_{\underline{n}} \mid \mathcal{F}_{n_{i}+m}^{(i)}\right)=U_{i}^{n_{i}+m}\left(U_{i}^{*}\right)^{n_{i}+m} U^{\underline{n}} f=U^{\underline{n}+m e_{i}}\left(U_{i}^{*}\right)^{k} f=0 \quad \text { for any } m \in \mathbb{N}^{*}, \underline{n} \in \mathbb{N}^{d} .
$$

For every $\underline{k}, \underline{h} \in \mathbb{N}^{d}$, we shall write $\underline{k} \preceq \underline{h}$ if for every $i \in\{1, \ldots, d\}$ we have $k_{i} \leq h_{i}$. For every $\underline{n} \in \overline{\mathbb{N}}^{d}$ and every $\underline{t} \in[0,1]^{d}$, we shall write $[\underline{n t}]:=\left(\left[n_{1} t_{1}\right], \ldots,\left[n_{d} t_{d}\right]\right)$, where $[\cdot]$ stands for the integer part.

Let $\left(U^{\underline{n}} f\right)_{\underline{n} \in \mathbb{N}^{d}}$ be a random field on $(\Omega, \mathcal{A}, \mu)$. For every $\underline{n}=\left(n_{1}, \ldots, n_{d}\right) \in \mathbb{N}^{d}$ and every $\underline{t}=\left(t_{1}, \ldots, t_{d}\right) \in[0,1]^{d}$ set

$$
\begin{aligned}
S_{\underline{n}, \underline{t}}(f) & :=\sum_{0 \preceq \underline{k} \preceq[\underline{n t}]} \prod_{i=1}^{d}\left(k_{i} \wedge\left(n_{i} t_{i}-1\right)-k_{i}+1\right) U^{\underline{k}} f, \\
\text { and } \quad T_{\underline{n}, \underline{t}}(f) & :=\frac{S_{\underline{n}, \underline{t}}(f)}{\left(\prod_{i=1}^{d} n_{i}\right)^{1 / 2}} .
\end{aligned}
$$


Theorem 1. Let $\left(U^{\underline{n}} f\right)_{\underline{n} \in \mathbb{N}^{d}}$ be a commuting stationary d-field of reverse martingale differences with $f \in L^{2}(\Omega, \mathcal{A}, \mu)$. Assume that one of the $U_{i}$ 's is ergodic. Then, the process $\left.\left(T_{\underline{n}, \underline{t}}(f)\right)_{\underline{t} \in[0,1]^{d}}\right)_{\underline{n} \in \mathbb{N}^{d}}$ converges in law in $C\left([0,1]^{d}\right)$ to $\left(\|f\|_{2} W_{\underline{t}}\right)_{\underline{t} \in[0,1]^{d}}$, where $\left(W_{\underline{t}}\right)_{\underline{t} \in[0,1]^{d}}$ is the standard d-dimensional brownian sheet.

Remark. Recall that $\left(W_{\underline{t}}\right)_{\underline{t} \in[0,1]^{d}}$ is the centered gaussian process characterized by $\mathbb{E}\left(W_{\underline{s}} W_{\underline{t}}\right)=\prod_{i=1}^{d}\left(s_{i} \wedge t_{i}\right)$. The ergodicity will be needed at the very end of the proof, in order to prove Lemma 4 below.

As usual we shall prove that result in two steps. The first one consists in proving tightness. The second one consists in proving convergence of the finite dimensional distributions.

3.1. Proof of the tightness. The tightness has been proved by Volný and Wang [20] in the case of martingale differences rather than reverse martingale differences. Their argument carry on to our setting provided that we have a maximal inequality of Cairoli's type for reverse martingale fields.

We shall just state the appropriate version of Cairoli's maximal inequality needed and refer to [20] for the proof of the tightness. We state it in the stationary case, but it holds in a more general setting.

Proposition 2 (Cairoli, [1]). Let $\left(U^{\underline{n}} f\right)_{n \in \mathbb{N}^{d}}$ be a commuting stationary d-field of reverse martingale differences with $f \in L^{p}(\Omega, \mathcal{A}, \mu), p>1$. Then, for every $\underline{n} \in \mathbb{N}^{d}$ we have

$$
\mathbb{E}\left(\max _{1 \preceq \underline{k} \preceq \underline{n}}\left|\sum_{0 \preceq \underline{i} \preceq \underline{k}} U^{\underline{i} f} f\right|^{p}\right) \leq 2^{d p}\left(\frac{p}{p-1}\right)^{d} \mathbb{E}\left(\left|\sum_{0 \preceq \underline{i} \preceq \underline{n}} U^{\underline{i}} f\right|^{p}\right)
$$

Proof. We explain how to derive the result from Cairoli's original result when $d=2$. Let $\underline{n} \in \mathbb{N}^{2}$. For every $0 \preceq \underline{k} \preceq \underline{n}$ write $g_{\underline{k}}:=U^{\underline{n}-\underline{k}} f$. Then, $\left(\sum_{0 \preceq \underline{i} \preceq \underline{k}} g_{\underline{i}}\right)_{0 \preceq \underline{k} \preceq \underline{n}}$ is a so-called orthomartingale. Moreover, we see that for every $0 \preceq \underline{k} \preceq \underline{n}$,

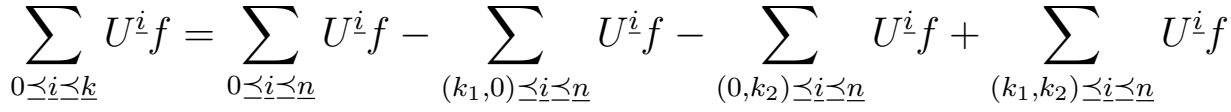

$$
\begin{aligned}
& =\sum_{0 \preceq \underline{i} \preceq \underline{n}} g_{\underline{i}}-\sum_{0 \preceq \underline{i} \preceq\left(n_{1}-k_{1}, 0\right)} g_{\underline{i}}-\sum_{0 \preceq \underline{i} \preceq\left(0, n_{2} k_{2}\right)} g_{\underline{i}}+\sum_{0 \preceq \underline{\imath} \preceq\left(n_{1}-k_{1}, n_{2}-k_{2}\right)} g_{\underline{i}} .
\end{aligned}
$$

3.2. Convergence of the finite dimensional distributions. We have to prove that for every $\left(\underline{t}_{k}\right)_{0 \leq k \leq L}$ with $\underline{t}_{k} \in[0,1]^{d}$,

$$
\left(T_{\underline{n}, \underline{t}_{0}}(f), \ldots, T_{\underline{n}, \underline{t}_{L}}(f)\right) \Rightarrow\left(W_{\underline{t}_{0}}(f), \ldots W_{\underline{t}_{L}}(f)\right)
$$

as $n_{1}, \ldots, n_{d} \rightarrow+\infty$. 
Note first that, if

$$
\widetilde{S}_{\underline{n}, \underline{t}}(f):=\sum_{0 \preceq \underline{k} \preceq[\underline{n} t]-\underline{1}} U^{\underline{k}} f \quad \text { and } \quad \widetilde{T}_{\underline{n}, \underline{t}}(f):=\frac{\widetilde{S}_{\underline{n}, \underline{t}}(f)}{\left(\prod_{i=1}^{d} n_{i}\right)^{1 / 2}},
$$

where $\underline{1}=(1, \ldots, 1)$, then $\left\|T_{\underline{n}, \underline{t}}(f)-\widetilde{T}_{\underline{n}, \underline{t}}(f)\right\|_{2} \rightarrow 0$ as $n_{1}, \ldots, n_{d} \rightarrow+\infty$. This follows easily from the fact that, using the reverse-martingale property,

$$
\left\|S_{\underline{n}, \underline{t}}(f)-\widetilde{S}_{\underline{n}, \underline{t}}(f)\right\|_{2}^{2} \leq\|f\|_{2}^{2}\left(\sum_{i=1}^{d} \prod_{j \in\{1, \ldots, d\}, j \neq i} n_{j}\right) .
$$

Hence, is suffices to prove (6) with $\widetilde{T}_{\underline{n}, \underline{t}_{i}}$ instead of $T_{\underline{n}, \underline{t}_{i}}$. Let $\left(a_{k}\right)_{0 \leq k \leq L}$ be $L+1$ real numbers. By the Cramer-Wold device, it suffices to prove that

$$
\sum_{k=0}^{L} a_{k} \widetilde{T}_{\underline{n}, \underline{t}_{k}}(f) \Rightarrow \sum_{k=0}^{L} a_{k} W_{\underline{t}_{k}}(f) .
$$

As a second simple remark, note that the sum $\sum_{k=0}^{L} a_{k} \widetilde{T}_{\underline{n}, \underline{t}_{k}}(f)$ can be written as a weighted sum over disjoint and adjacent rectangles. Hence, it suffices to prove the convergence in distribution for such rectangles.

We shall make the proof when $d=2$, the general case can be proved by induction. Let $t_{0}=0<t_{1}<\cdots<t_{K} \leq 1$ and $s_{0}=0<s_{1}<\cdots<s_{K} \leq 1$. Let also $\left(a_{k, \ell}\right)_{1 \leq k, \ell \leq K}$ be real numbers. From the remarks above, it suffices to prove that

$$
\begin{aligned}
V_{n_{1}, n_{2}}= & \frac{1}{\sqrt{n_{1} n_{2}}} \sum_{k=1}^{K} \sum_{\ell=1}^{K} a_{k, \ell} \sum_{i=\left[n_{1} t_{k-1}\right]}^{\left[n t_{k}\right]-1} \sum_{j=\left[n_{2} s_{\ell-1}\right]}^{\left[n s_{\ell}\right]-1} U_{1}^{i} U_{2}^{j}(f) \\
& \Rightarrow \sum_{k=1}^{K} \sum_{\ell=1}^{K} a_{k, \ell}\left(W_{\left(t_{k}, s_{\ell}\right)}(f)+W_{\left(t_{k-1}, s_{\ell-1}\right)}(f)-W_{\left(t_{k}, s_{\ell-1}\right)}(f)-W_{\left(t_{k-1}, s_{\ell}\right)}(f)\right) .
\end{aligned}
$$

as $n_{1}, n_{2} \rightarrow \infty$. Notice that the random variable on right hand is distributed according to $\mathcal{N}(0, \Gamma)$, with

$$
\Gamma=\|f\|_{2}^{2} \sum_{k=1}^{K} \sum_{\ell=1}^{K} a_{k, \ell}^{2}\left(t_{k}-t_{k-1}\right)\left(s_{\ell}-s_{\ell-1}\right) .
$$

Clearly, it suffices to prove the desired convergence in distribution when $n_{1}, n_{2} \rightarrow+\infty$ along any sequence $\left(m_{r}, n_{r}\right)_{r \geq 1}$. Hence, let us fix a sequence $\left(m_{r}, n_{r}\right)_{r \geq 1}$ such that $m_{r}, n_{r} \rightarrow+\infty$ as $r \rightarrow+\infty$. It remains to prove that

$$
V_{r}=\frac{1}{\sqrt{m_{r} n_{r}}} \sum_{k=1}^{K} \sum_{\ell=1}^{K} a_{k, \ell} \sum_{i=\left[m_{r} t_{k-1}\right]}^{\left[m_{r} t_{k}\right]-1} \sum_{j=\left[n_{r} s_{\ell-1}\right]}^{\left[n_{r} s_{\ell}\right]-1} U_{1}^{i} U_{2}^{j}(f) \Rightarrow \mathcal{N}(0, \Gamma)
$$

Proof of (7). Since one of the $U_{i}^{\prime} s$ is assumed to be ergodic, let us assume that $U_{2}$ is. 
We will apply the following result of McLeish as stated in Hall and Heyde [12] (see Theorem 3.6 p. 77). This theorem is stated for an array of martingale differences, but a simple change of time gives the next proposition. We first mention what we mean by an array of reverse martingale differences.

Definition 3. Let $\left(\left(X_{r, k}\right)_{0 \leq k \leq p_{r}-1}\right)_{r \geq 1}$ be an array of variables and $\left(\left(\mathcal{G}_{r, k}\right)_{0 \leq k \leq p_{r}-1}\right)_{r \geq 1}$ be an array of $\sigma$-algebras, such that for every $r \geq 1,\left(\mathcal{G}_{r, k}\right)_{0 \leq k \leq p_{r}-1}$ is decreasing. We say that $\left(\left(X_{r, k},\left(\mathcal{G}_{r, k}\right)_{0 \leq k \leq p_{r}-1}\right)_{r \geq 1}\right.$ is an array of reverse martingale differences if $X_{r, k}$ is $\mathcal{G}_{r, k}$-measurable for every $r \geq 1$ and $1 \leq k \leq p_{r}$ and if $\mathbb{E}\left(X_{r, k} \mid \mathcal{G}_{r, k+1}\right)=0$ for every $r \geq 1$ and every $0 \leq k \leq p_{r}-2$.

Proposition 3 (McLeish). Let $\left(\left(X_{r, k},\left(\mathcal{G}_{r, k}\right)_{0 \leq k \leq p_{r}-1}\right)_{r \geq 1}\right.$ be an array of reverse martingale differences in $L^{2}$. Assume that

(i) $\sup _{0 \leq k \leq p_{r}-1}\left|X_{r, k}\right| \stackrel{P}{\rightarrow} 0$;

(ii) $\sup _{0 \leq k \leq p_{r}-1} \mathbb{E}\left(X_{r, k}^{2}\right)<\infty$;

(iii) $\sum_{k=0}^{p_{r}-1} X_{r, k}^{2} \stackrel{P}{\rightarrow} V$ for some $V \geq 0$;

Then, $\sum_{0 \leq k \leq p_{r}-1} X_{r, k}$ converges in distribution to $\mathcal{N}(0, V)$.

To apply this proposition, we write

$$
\frac{1}{\sqrt{m_{r} n_{r}}} \sum_{i=0}^{m_{r}-1} \sum_{k=1}^{K} \mathbf{1}_{\left\{\left[m_{r} t_{k-1}\right] \leq i \leq\left[m_{r} t_{k}\right]-1\right\}}\left(\sum_{\ell=1}^{K} a_{k, \ell} \sum_{j=\left[n_{r} s_{\ell-1}\right]}^{\left[n_{r} s_{\ell}\right]-1} U_{1}^{i} U_{2}^{j}(f)\right):=\sum_{i=0}^{m_{r}-1} Z_{r, i} .
$$

Note that $Z_{r, i}$ is a reverse martingale difference with respect to $\mathcal{G}_{r, i}=\mathcal{F}_{i}^{(1)}$. We shall now prove that $\left(Z_{r, i}\right)_{0 \leq i \leq m_{r}-1}$ satisfies $(i),(i i)$ and (iii) of Proposition 3 (with $V=\Gamma$ for $(i i i))$.

Proof of $(i)$ and $(i i)$. We have

$$
\sup _{0 \leq i \leq m_{r}-1}\left|Z_{r, i}\right| \leq\left(\sup _{1 \leq k \leq K} \sum_{\ell=1}^{K}\left|a_{k, \ell}\right|\right) \sup _{1 \leq \ell \leq K} \sup _{0 \leq i \leq m_{r}-1} \frac{1}{\sqrt{m_{r} n_{r}}}\left|\sum_{j=\left[n_{r} s_{\ell-1}\right]}^{\left[n_{r} s_{\ell}\right]-1} U_{1}^{i} U_{2}^{j}(f)\right| .
$$

Hence we just have to prove that for every $\varepsilon>0$, and every $1 \leq \ell \leq K$,

$$
\gamma_{\ell, \varepsilon, r}:=\mu\left(\sup _{0 \leq i \leq m_{r}-1} \frac{1}{\sqrt{m_{r} n_{r}}}\left|\sum_{j=\left[n_{r} s_{\ell-1}\right]}^{\left[n_{r} s_{\ell}\right]-1} U_{1}^{i} U_{2}^{j}(f)\right| \geq \varepsilon\right) \underset{r \rightarrow+\infty}{\longrightarrow \rightarrow} 0 .
$$

Using the stationarity and Markov inequality, we obtain that

$$
\begin{aligned}
\gamma_{j, \varepsilon, r} & \leq m_{r} \mu\left(\frac{1}{\sqrt{m_{r} n_{r}}} \sum_{j=\left[n_{r} s_{\ell-1}\right]}^{\left[n_{r} s_{\ell}\right]-1} U_{2}^{j}(f) \mid \geq \varepsilon\right) \\
& \leq \frac{1}{\varepsilon^{2}} \mathbb{E}\left(\left(\frac{1}{\sqrt{n_{r}}} \sum_{j=\left[n_{r} s_{\ell-1}\right]}^{\left[n_{r} s_{\ell}\right]-1} U_{2}^{j}(f)\right)^{2} \mathbf{1}_{\left|\sum_{j=\left[n_{r} s_{\ell-1}\right]}^{\left[n_{r} s_{\ell}\right]-1} U_{2}^{j}(f)\right|>\sqrt{m_{r} n_{r} \varepsilon}}\right) .
\end{aligned}
$$


Notice that $\left(U_{2}^{i} f\right)_{0 \leq 1 \leq m_{r}-1}$ is a stationary sequence of reverse martingale differences. Now, it is well-known (using stationarity, truncation and Burkholder inequality) that the family

$$
\left(Y_{r, \ell}\right)_{r \geq 1}:=\left(\left(\frac{1}{\sqrt{n_{r}}} \sum_{j=\left[n_{r} s_{\ell-1}\right]}^{\left[n_{r} s_{\ell}\right]-1} U_{2}^{j}(f)\right)^{2}\right)_{r \geq 1}
$$

is uniformly integrable, and $(i)$ easily follows.

In the same way, $(\mathrm{ii})$ can be proved by using the stationarity and the fact that $\left(Y_{r, \ell}\right)_{r \geq 1}$ is bounded in $L^{1}$.

Proof of $($ iii $)$. This is the difficult part. It suffices to prove that

$$
\lim _{r \rightarrow \infty}\left\|\sum_{i=0}^{m_{r}-1} Z_{r, i}^{2}-\Gamma\right\|_{1}=0
$$

Now

$$
Z_{r, i}^{2}=\sum_{k=1}^{K} \frac{1}{m_{r}} \mathbf{1}_{\left\{\left[m_{r} t_{k-1}\right] \leq i \leq\left[m_{r} t_{k}\right]-1\right\}}\left(\sum_{\ell=1}^{K} a_{k, \ell} \frac{1}{\sqrt{n}_{r}} \sum_{j=\left[n_{r} s_{\ell-1}\right]}^{\left[n_{r} s_{\ell}\right]-1} U_{1}^{i} U_{2}^{j}(f)\right)^{2} .
$$

Hence, it suffices to prove that

$$
\lim _{r \rightarrow \infty} \sum_{k=1}^{K}\left\|\frac{1}{m_{r}} \sum_{i=\left[m_{r} t_{k-1}\right]}^{\left[m_{r} t_{k}\right]-1}\left(\sum_{\ell=1}^{K} a_{k, \ell} \frac{1}{\sqrt{n}_{r}} \sum_{j=\left[n_{r} s_{\ell-1}\right]}^{\left[n_{r} s_{\ell}\right]-1} U_{1}^{i} U_{2}^{j}(f)\right)^{2}-\gamma_{k}\right\|_{1}=0
$$

where $\gamma_{k}=\|f\|_{2}^{2} \sum_{\ell=1}^{K} a_{k, \ell}^{2}\left(t_{k}-t_{k-1}\right)\left(s_{\ell}-s_{\ell-1}\right)$. By stationarity, it suffices to prove that, for each $k \in\{1, \ldots, K\}$,

$$
\lim _{r \rightarrow \infty}\left\|\frac{1}{m_{r}} \sum_{i=0}^{\left[m_{r} t_{k}\right]-\left[m_{r} t_{k-1}\right]-1}\left(\sum_{\ell=1}^{K} a_{k, \ell} \frac{1}{\sqrt{n_{r}}} \sum_{j=\left[n_{r} s_{\ell-1}\right]}^{\left[n_{r} s_{\ell}\right]-1} U_{1}^{i} U_{2}^{j}(f)\right)^{2}-\gamma_{k}\right\|_{1}=0 .
$$

Setting $u_{r}=\left[m_{r} t_{k}\right]-\left[m_{r} t_{k-1}\right]$, this is equivalent to

(8) $\lim _{r \rightarrow \infty}\left\|\frac{1}{u_{r}} \sum_{i=0}^{u_{r}-1}\left(\sum_{\ell=1}^{K} a_{k, \ell} \frac{1}{\sqrt{n}_{r}} \sum_{j=\left[n_{r} s_{\ell-1}\right]}^{\left[n_{r} s_{\ell}\right]-1} U_{1}^{i} U_{2}^{j}(f)\right)^{2}-\right\| f\left\|_{2}^{2} \sum_{\ell=1}^{K} a_{k, \ell}^{2}\left(s_{\ell}-s_{\ell-1}\right)\right\|_{1}=0$.

In order to prove (8), let us admit the following lemma for a while.

Lemma 4. Let $\varepsilon>0$. If $U_{2}$ is ergodic, there exist integers $v \geq 1$ (large enough) and $p(v)$ (large enough), such that for every $n \geq p(v)$

$$
\left\|\frac{1}{v} \sum_{i=0}^{v-1}\left(\frac{1}{\sqrt{n}} \sum_{\ell=1}^{K} a_{k, \ell} \sum_{j=\left[n s_{\ell-1}\right]}^{\left[n s_{\ell}\right]-1} U_{1}^{i} U_{2}^{j}(f)\right)^{2}-\Delta_{k}\right\|_{1}<\varepsilon,
$$


where $\Delta_{k}:=\|f\|_{2}^{2} \sum_{\ell=1}^{K} a_{k, \ell}^{2}\left(s_{\ell}-s_{\ell-1}\right)$.

Let

$$
F_{r, i}=\sum_{\ell=1}^{K} a_{k, \ell} \frac{1}{\sqrt{n}_{r}} \sum_{j=\left[n_{r} s_{\ell-1}\right]}^{\left[n_{r} s_{\ell}\right]-1} U_{1}^{i} U_{2}^{j}(f) .
$$

Let $r \geq 1$, such that $u_{r} \geq v$ and write $u_{r}=v q_{r}+t_{r}$, with $q_{r} \geq 1$ and $t_{r} \in\{0, \ldots, v-1\}$. We have that

$$
\frac{1}{u_{r}} \sum_{i=0}^{u_{r}-1} F_{r, i}^{2}-\Delta_{k}=\frac{v}{u_{r}} \sum_{k=0}^{q_{r}-1}\left(\frac{1}{v} \sum_{i=k v}^{(k+1) v-1} F_{r, i}^{2}-\Delta_{k}\right)+\frac{1}{u_{r}} \sum_{i=v q_{r}}^{u_{r}-1} F_{r, i}^{2}-\frac{t_{r} \Delta_{k}}{u_{r}}
$$

By stationarity, and Lemma 4, we see that, for $n_{r} \geq p(v)$

$$
\begin{aligned}
\left\|\frac{v}{u_{r}} \sum_{k=0}^{q_{r}-1}\left(\frac{1}{v} \sum_{i=k v}^{(k+1) v-1} F_{r, i}^{2}-\Delta_{k}\right)\right\|_{1} & \|\| \frac{1}{v} \sum_{i=0}^{v-1}\left(\frac{1}{\sqrt{n_{r}}} \sum_{\ell=1}^{K} a_{k, \ell} \sum_{j=\left[n_{r} s_{\ell-1}\right]}^{\left[n_{r} s_{\ell}\right]-1} U_{1}^{i} U_{2}^{j}(f)\right)^{2}-\Delta_{k} \|_{1}<\varepsilon .
\end{aligned}
$$

On another hand, for a fixed $v$, one has

$$
\lim _{r \rightarrow \infty}\left\|\frac{1}{u_{r}} \sum_{i=v q_{r}}^{u_{r}-1} F_{r, i}^{2}-\frac{t_{r} \Delta_{k}}{u_{r}}\right\|_{1}=0 .
$$

From (10), (11) and (12), we see that (8) holds. This completes the proof of (iii).

Proof of Lemma 4 The proof relies on the following convergence in law.

Lemma 5. Let $v \geq 1$. The sequence of random vectors

$$
\left(\frac{1}{\sqrt{n}} \sum_{\ell=1}^{K} a_{k, \ell} \sum_{j=\left[n s_{\ell-1}\right]}^{\left[n s_{\ell}\right]-1} U_{1}^{i} U_{2}^{j}(f)\right)_{1 \leq i \leq v}
$$

converges in distribution to $\left(N_{i}\right)_{1 \leq i \leq v}$, where the $N_{i}$ 's are iid with common distribution $\mathcal{N}\left(0,\|f\|_{2}^{2} \sum_{\ell=1}^{K} a_{k, \ell}^{2}\left(s_{\ell}-s_{\ell-1}\right)\right)$.

Lemma 4 follows easily from Lemma 5 , a truncation argument and the law of large numbers in $L^{1}$ for $\left(N_{i}^{2}\right)_{1 \leq i \leq v}$ (with $v \rightarrow+\infty$ ).

Lemma 5 can be proved by applying Proposition 3, but it is shorter to notice that it is a consequence of the WIP for stationary and ergodic $\mathbb{R}^{v}$-valued reverse martingale differences (note that it is the only place where we use the ergodicity of $U_{2}$ ). Indeed, 
letting $V(f)=\left(U_{1}^{1}(f), \ldots, U_{1}^{v}(f)\right)^{\prime}$, it follows from the WIP that

$$
\left(\frac{1}{\sqrt{n}} \sum_{j=0}^{\left[n s_{1}\right]-1} V\left(U_{2}^{j}(f)\right), \ldots, \frac{1}{\sqrt{n}} \sum_{j=\left[n s_{K-1}\right]}^{\left[n s_{K}\right]-1} V\left(U_{2}^{j}(f)\right)\right)
$$

converges in distribution to $\left(G_{1}, \ldots, G_{K}\right)$, where the $G_{\ell}$ 's are independent Gaussian random vectors with respective covariance matrix $\left(s_{\ell}-s_{\ell-1}\right) \mathbb{E}\left(V(f) V(f)^{\prime}\right)$. Now since $\mathbb{E}\left(U_{1}^{i}(f) U_{1}^{j}(f)\right)=0$ if $i \neq j$, we see that $\mathbb{E}\left(V(f) V(f)^{\prime}\right)=\|f\|_{2}^{2} \operatorname{Id}_{v}$, where $\operatorname{Id}_{v}$ is the identity $v \times v$ matrix. Lemma 5 follows straightforwardly.

\section{ReVERSE martingale approximation}

We shall consider again a completely commuting family of (non invertible) measure preserving transformations $T_{1}, \ldots, T_{d}$.

In all that section we assume the following property

$$
\left\|\left(U_{i}^{*}\right)^{n} f\right\|_{2} \underset{n \rightarrow+\infty}{\longrightarrow} 0 \text { for any } i \in\{1, \ldots, d\} \text {, and any } f \in L^{2}(\Omega, \mathcal{A}, \mu) \text { with } \mathbb{E}(f)=0 \text {. }
$$

This property is equivalent to the fact that each $T_{i}$ is exact (see Definition 4.14 in Walters [21]).

We shall now prove a (reverse) martingale approximation result under a condition in the spirit of Hannan. For $f \in L^{2}(\mu)$ set

$$
\begin{aligned}
\|f\|_{\mathcal{X}_{2}}: & =\sum_{n_{1}, \ldots, n_{d} \in \mathbb{N}}\left\|\prod_{i=1}^{d}\left(U_{i}^{n_{i}}\left(U_{i}^{*}\right)^{n_{i}}-U_{i}^{n_{i}+1}\left(U_{i}^{*}\right)^{n_{i}+1}\right) f\right\|_{2} \\
& =\sum_{n_{1}, \ldots, n_{d} \in \mathbb{N}}\left\|\prod_{i=1}^{d}\left(\left(U_{i}^{*}\right)^{n_{i}}-U_{i}\left(U_{i}^{*}\right)^{n_{i}+1}\right) f\right\|_{2},
\end{aligned}
$$

and

$$
\mathcal{X}_{2}:=\left\{f \in L^{2}(\mu):\|f\|_{\mathcal{X}_{2}}<\infty\right\}
$$

It is not hard to prove as in the one-dimensional case (see for instance the proof of Proposition 12 in [5]) that a sufficient condition for $f$ to be in $\mathcal{X}_{2}$ is that

$$
\sum_{n_{1}, \ldots, n_{d} \in \mathbb{N}} \frac{\left\|\left(U_{1}^{*}\right)^{n_{1}} \cdots\left(U_{d}^{*}\right)^{n_{d}} f\right\|_{2}}{\left(n_{1} \cdots n_{d}\right)^{1 / 2}}<\infty .
$$

We first prove a maximal inequality. Its statement, as well as its proof, are analogous to Lemma 5.2 of [20].

Lemma 6. Let $f \in \mathcal{X}_{2}$. Then,

$$
\mathbb{E}\left(\max _{1 \preceq \underline{k} \preceq \underline{n}}\left|\sum_{0 \preceq \underline{i} \leq \underline{k}} U^{\underline{i}} f\right|^{2}\right) \leq 2^{3 d}\left(n_{1} \cdots n_{d}\right)\|f\|_{\mathcal{X}_{2}}^{2} .
$$


Proof. Let $f \in \mathcal{X}_{2}$. Using that for every $i \in\{1, \ldots, d\},\left\|\left(U_{i}^{*}\right)^{n} f\right\|_{2} \longrightarrow 0$ as $n \rightarrow \infty$, we obtain the following orthogonal decomposition

$$
f=\sum_{m_{1}, \ldots, m_{d} \in \mathbb{N}} \prod_{i=1}^{d}\left(U_{i}^{m_{i}}\left(U_{i}^{*}\right)^{m_{i}}-U_{i}^{m_{i}+1}\left(U_{i}^{*}\right)^{m_{i}+1}\right) f:=\sum_{\underline{m} \in \mathbb{N}^{d}} f_{\underline{m}} .
$$

Then, clearly

$$
\max _{1 \preceq \underline{k} \preceq \underline{n}}\left|\sum_{0 \preceq \underline{\imath} \preceq \underline{k}} U^{\underline{i}} f\right| \leq \sum_{\underline{m} \in \mathbb{N}^{d}} \max _{1 \preceq \underline{k} \preceq \underline{n}}\left|\sum_{0 \underline{i} \underline{\underline{k}} \underline{\underline{k}}} U^{\underline{i}} f_{\underline{m}}\right|
$$

Now, for every $\underline{m}=\left(m_{1}, \ldots, m_{d}\right) \in \mathbb{N}^{d},\left(U^{\underline{i}} f_{\underline{m}}\right)_{i \in \mathbb{N}^{d}}$ is a commuting stationary $d$ field of reverse martingale differences associated with $f_{\underline{m}} \in L^{2}\left(\Omega, \mathcal{A}_{\underline{m}}, \mu\right)$, where $\mathcal{A}_{\underline{m}}=$ $T_{1}^{-m_{1}} \circ \cdots \circ T_{d}^{-m_{d}}(\mathcal{A})$. Hence, the result follows from Proposition 2.

We shall also need the following lemma.

Lemma 7. Let $T_{1}$ be a measure preserving transformation and $U_{1}$ the associated Koopman operator. Let $f, g \in L^{2}(\Omega, \mathcal{A}, \mu)$ be such that for $h \in\{f, g\}$,

$$
\sum_{n \geq 0}\left\|\left(\left(U_{1}^{*}\right)^{n}-U_{1}\left(U_{1}^{*}\right)^{n+1}\right) h\right\|_{2}<\infty .
$$

Then, $\sum_{k \in \mathbb{Z}}\left|\mathbb{E}\left(U_{1}^{k^{+}} f U_{1}^{k^{-}} g\right)\right|<\infty$, where $k^{+}=\max \{0, k\}$ and $k^{-}=\max \{0,-k\}$. Moreover, writing $\tilde{f}:=\sum_{k \geq 0}\left(\left(U_{1}^{*}\right)^{k}-U_{1}\left(U_{1}^{*}\right)^{k+1}\right) f$ and $\tilde{g}:=\sum_{k \geq 0}\left(\left(U_{1}^{*}\right)^{k}-U_{1}\left(U_{1}^{*}\right)^{k+1}\right) g$,

$$
\mathbb{E}(\tilde{f} \tilde{g})=\sum_{k \in \mathbb{Z}} \mathbb{E}\left(U_{1}^{k^{+}} f U_{1}^{k^{-}} g\right) .
$$

Proof. The absolute convergence of the series will follow from the proof. Hence, we only prove (18). For every $f \in L^{2}(\Omega, \mathcal{A}, \mu)$ such that $\mathbb{E}(f)=0$, using that $\left\|\left(U_{1}^{*}\right)^{n} f\right\|_{2} \underset{n \rightarrow+\infty}{\longrightarrow} 0$, we have

$$
f=\sum_{n \in \mathbb{N}}\left(U_{1}^{n}\left(U_{1}^{*}\right)^{n}-U_{1}^{n+1}\left(U_{1}^{*}\right)^{n+1}\right) f,
$$

where the summands are orthogonal.

Let

$$
A_{k, \ell}(f, g)=\mathbb{E}\left(\left(\left(\left(U_{1}^{*}\right)^{k}-U_{1}\left(U_{1}^{*}\right)^{k+1}\right) f\right)\left(\left(\left(U_{1}^{*}\right)^{\ell}-U_{1}\left(U_{1}^{*}\right)^{\ell+1}\right) g\right)\right)
$$

Using (17) to permute $\mathbb{E}$ and $\sum$, we have (with absolute convergence)

$$
\mathbb{E}(\tilde{f} \tilde{g})=\sum_{k, \ell \in \mathbb{N}} A_{k, \ell}(f, g)=\sum_{k, \ell: k>\ell} A_{k, \ell}(f, g)+\sum_{k, \ell: k<\ell} A_{k, \ell}(f, g)+\sum_{k, \ell: k=\ell} A_{k, \ell}(f, g) .
$$

The first two sums on right hand are symmetric one from the other, hence we shall deal only with the second one. Since $\left.\mathbb{E}\left(\left(\left(U_{1}^{*}\right)^{k}-U_{1}\left(U_{1}^{*}\right)^{k+1}\right) f\right)\left(U_{1}\left(U_{1}^{*}\right)^{\ell+1} g\right)\right)=0$ for 
$\ell \geq k$

$$
\begin{aligned}
\sum_{k \in \mathbb{N}} \sum_{\ell>k} A_{k, \ell}(f, g) & =\sum_{k \in \mathbb{N}} \sum_{m \in \mathbb{N}^{*}} \mathbb{E}\left(\left(\left(U_{1}^{k}\left(U_{1}^{*}\right)^{k}-U_{1}^{k+1}\left(U_{1}^{*}\right)^{k+1}\right) f\right)\left(U_{1}^{*}\right)^{m} g\right) \\
& =\sum_{m \in \mathbb{N}^{*}} \mathbb{E}\left(U_{1}^{m} f g\right)
\end{aligned}
$$

where we have used (19). In the same way

$$
\sum_{k \in \mathbb{N}} A_{k, k}(f, g)=\sum_{k \in \mathbb{N}} \mathbb{E}\left(\left(\left(U_{1}^{k}\left(U_{1}^{*}\right)^{k}-U_{1}^{k+1}\left(U_{1}^{*}\right)^{k+1}\right) f\right) g\right)=\mathbb{E}(f g) .
$$

Theorem 8. Let $f \in \mathcal{X}_{2}$. Then, there exists a commuting stationary d-field of reverse martingale differences $\left(U^{\underline{n}}(d)\right)_{\underline{n} \in \mathbb{N}^{d}}$ with $d \in L^{2}(\mu)$ such that

$$
\mathbb{E}\left(\max _{0 \preceq \underline{k} \preceq \underline{n}}\left|\sum_{0 \preceq \underline{i} \preceq \underline{k}} U^{i} f-U^{\underline{i}} d\right|^{2}\right)=o\left(n_{1} \cdots n_{d}\right), \quad \text { as } n_{1}, \ldots, n_{d} \rightarrow+\infty,
$$

and $\|d\|_{2}^{2}=\sum_{\underline{n} \in \mathbb{N}^{d}} \mathbb{E}\left(U \underline{n}^{+} f U^{\underline{n}} f\right)$, where we use the notations $\underline{n}^{+}=\left(n_{1}^{+}, \ldots, n_{d}^{+}\right)$and $\underline{n}^{-}=\left(n_{1}^{-}, \ldots, n_{d}^{-}\right)$.

Proof. By (19) we see that $\|\cdot\|_{\mathcal{X}_{2}}$ is definite on $\mathcal{X}_{2}$ hence that it is a norm. Moreover, $\left(\mathcal{X}_{2},\|\cdot\|_{\mathcal{X}_{2}}\right)$ is a Banach space.

Let $i \in\{1, \ldots, d\}$. We easily see that $\left\|\left(U_{i}^{*}\right)^{n} f\right\|_{\mathcal{X}_{2}} \longrightarrow 0$ as $n \rightarrow+\infty$. Hence, $U_{i}^{*}$ is mean ergodic on $\mathcal{X}_{2}$ (with no fixed points), that is

$$
\mathcal{X}_{2}={\overline{\left(I-U_{i}^{*}\right) \mathcal{X}_{2}}}^{\mathcal{X}_{2}} .
$$

Then, it follows that

$$
\mathcal{X}_{2}=\bar{\prod}_{1 \leq i \leq d}\left(I-U_{i}^{*}\right) \mathcal{X}_{2}{ }^{\mathcal{X}_{2}}
$$

Define a linear operator $\mathcal{D}$ on $\mathcal{X}_{2}$ by setting

$$
\mathcal{D} f:=\sum_{\underline{n} \in \mathbb{N}^{d}} \prod_{i=1}^{d}\left(\left(U_{i}^{*}\right)^{n_{i}}-U_{i}\left(U_{i}^{*}\right)^{n_{i}+1}\right) f .
$$

Let us observe that if $f=\prod_{1 \leq i \leq d}\left(I-U_{i}^{*}\right) g$ with $g \in \mathcal{X}_{2}$, then $\mathcal{D} f=\prod_{1 \leq i \leq d}\left(I-U_{i} U_{i}^{*}\right) g$.

Obviously,

$$
\|\mathcal{D}(f)\|_{2} \leq\|f\|_{\mathcal{X}_{2}} .
$$

Let us prove (20) with $d=\mathcal{D}(f)$. Let us admit for a while that (20) holds whenever $f$ belongs to $\prod_{1 \leq i \leq d}\left(I-U_{i}^{*}\right) \mathcal{X}_{2}$. Let us show then that (20) holds for every $f \in \mathcal{X}_{2}$. 
Let $f \in \mathcal{X}_{2}$. Let $\varepsilon>0$. By (21), there exists $g \in \mathcal{X}_{2}$ such that

$$
\left\|f-\prod_{1 \leq i \leq d}\left(I-U_{i}^{*}\right) g\right\|_{\mathcal{X}_{2}}<\varepsilon .
$$

For every $\underline{n} \in \mathbb{N}^{d}$, we have, setting $\tilde{g}:=\prod_{1 \leq i \leq d}\left(I-U_{i}^{*}\right) g$,

$$
\begin{aligned}
\left|\sum_{0 \preceq \underline{k} \preceq \underline{n}}\left(U^{\underline{k}} f-U^{\underline{k}} \mathcal{D} f\right)\right| \leq \mid \sum_{0 \preceq \underline{k} \preceq \underline{n}}\left(U^{\underline{k}} f-U^{\underline{k} \tilde{g})} \mid\right. \\
\quad+\left|\sum_{0 \preceq \underline{k} \preceq \underline{n}}\left(U^{\underline{k}} h-U^{\underline{k}} \mathcal{D} \tilde{g}\right)\right|+\left|\sum_{0 \preceq \underline{k} \preceq \underline{n}}\left(U^{\underline{k}} \mathcal{D}(f-\tilde{g})\right)\right| .
\end{aligned}
$$

Using (15) to deal with the first term above and (22) and (5) to deal with the third term, and since we admit for the moment that (20) holds for $\tilde{g}$, we infer that

$$
\limsup _{n_{1}, \ldots, n_{d} \rightarrow+\infty} \frac{1}{n_{1} \cdots n_{d}} \mathbb{E}\left(\max _{1 \preceq \underline{k} \preceq \underline{n}}\left|\sum_{0 \preceq \underline{i} \preceq \underline{k}} U^{\underline{i}} f-U^{\underline{i}} d\right|^{2}\right) \leq C \varepsilon,
$$

and (20) follows by letting $\varepsilon \rightarrow 0$.

It remains to deal with the case where $f=\prod_{1 \leq i \leq d}\left(I-U_{i}^{*}\right) g$, for some $g \in \mathcal{X}_{2}$.

To do so we use the following simple identity (see also Gordin [9], Proposition 1):

$$
\text { for } i \in\{1, \ldots, d\}, \quad I-U_{i}^{*}=I-U_{i} U_{i}^{*}+\left(U_{i}-I\right) U_{i}^{*} .
$$

Let $f=\prod_{1 \leq i \leq d}\left(I-U_{i}^{*}\right) g$ with $g \in \mathcal{X}_{2}$. We have

$$
\begin{aligned}
f & =\mathcal{D} f+\sum_{\mathcal{E} \subset\{1, \ldots, d\}, \mathcal{E} \neq \emptyset} \prod_{i \in \mathcal{E}^{c}}\left(I-U_{i} U_{i}^{*}\right) \prod_{j \in \mathcal{E}}\left(U_{j}-I\right) U_{j}^{*} g \\
& =\mathcal{D} f+h .
\end{aligned}
$$

The proof relies on the fact that the remainder in (23) (i.e. $h$ ) behaves like a coboundary in some "directions" and like a sum of reverse martingale differences in the other "directions". For the sake of simplicity, we only prove the results for $d=2$, but the general case can be handled similarly.

We have

$$
f-\mathcal{D} f=\left(I-U_{1} U_{1}^{*}\right)\left(U_{2}-I\right) U_{2}^{*} g+\left(I-U_{2} U_{2}^{*}\right)\left(U_{1}-I\right) U_{1}^{*} g+\left(U_{1}-I\right)\left(U_{2}-I\right) U_{1}^{*} U_{2}^{*} g .
$$

For every $0 \leq k_{1} \leq n_{1}$ and every $0 \leq k_{2} \leq n_{2}$, we have

$$
\sum_{0 \leq i_{1} \leq k_{1}} U_{1}^{i_{1}} \sum_{0 \leq i_{2} \leq k_{2}} U_{2}^{i_{2}}\left(I-U_{2} U_{2}^{*}\right)\left(U_{1}-I\right) U_{1}^{*} g=\left(U_{1}^{k_{1}}-I\right) \sum_{0 \leq i_{2} \leq k_{2}} U_{1}^{i_{1}}\left(I-U_{2} U_{2}^{*}\right) U_{1}^{*} g .
$$


Define $Z_{k_{1}, n_{2}}:=\max _{1 \leq k_{2} \leq n_{2}}\left|\sum_{0 \leq i_{2} \leq k_{2}} U_{2}^{i_{2}}\left(I-U_{2} U_{2}^{*}\right) U_{1}^{k_{1}} U_{1}^{*} g\right|$. For every $A>0$, we have

$$
\begin{aligned}
\max _{1 \leq k_{1} \leq n_{1}} \max _{1 \leq k_{2} \leq n_{2}}\left|\sum_{0 \leq i_{1} \leq k_{1}} U_{1}^{i_{1}} \sum_{0 \leq i_{2} \leq k_{2}} U_{2}^{i_{2}}\left(I-U_{2} U_{2}^{*}\right)\left(U_{1}-I\right) U_{1}^{*} g\right|^{2} \\
\leq 2\left|Z_{0, n_{2}}\right|^{2}+2 n_{2} A^{2}+2 \sum_{0 \leq k_{1} \leq n_{1}}\left|Z_{k_{1}, k_{2}} \mathbf{1}_{\left\{\left|Z_{k_{1}, n_{2}}\right| \geq A \sqrt{n_{2}}\right\}}\right|^{2}
\end{aligned}
$$

and, by stationarity

$$
\begin{array}{r}
\mathbb{E}\left(\max _{1 \leq k_{2} \leq n_{2}} \max _{1 \leq k_{1} \leq n_{1}}\left|\sum_{0 \leq i_{1} \leq k_{1}} U_{1}^{i_{1}} \sum_{0 \leq i_{2} \leq k_{2}} U_{2}^{i_{2}}\left(I-U_{2} U_{2}^{*}\right)\left(U_{1}-I\right) U_{1}^{*} g\right|^{2}\right) \\
\leq 2 \mathbb{E}\left(\left|Z_{0, n_{2}}\right|^{2}\right)+2 n_{2} A^{2}+2 n_{1} \mathbb{E}\left(\left|Z_{0, n_{2}} \mathbf{1}_{\left\{\left|Z_{0, n_{2}}\right| \geq A \sqrt{n_{2}}\right\}}\right|^{2}\right) .
\end{array}
$$

Since, $\left(Z_{0, n_{2}}^{2} / n_{2}\right)_{n_{2} \geq 1}$ is uniformly integrable, it follows that

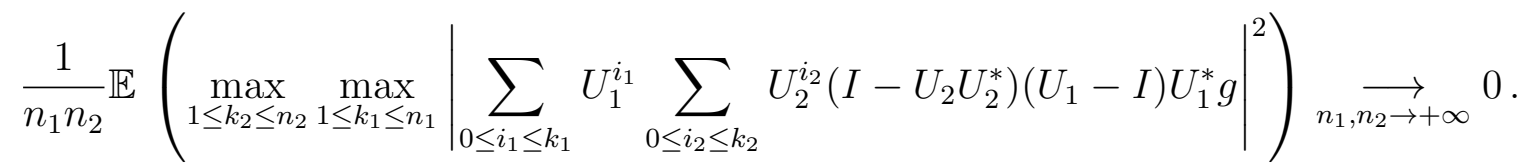

We may deal similarly with the sum associated with the term $\left(I-U_{1} U_{1}^{*}\right)\left(U_{2}-I\right) U_{2}^{*} g$. To deal with the sum associated with the term $\left.\left.\left(U_{1}-I\right)-U_{2}-I\right) U_{1}^{*}\right) U_{2}^{*} g$ is somehow easier.

To finish the proof of the theorem, it remains to identify $\|\mathcal{D} f\|_{2}^{2}$. But, this follows by applying inductively Lemma 7, noticing that

$$
\sum_{\underline{n} \in \mathbb{N}^{d}} \prod_{i=1}^{d}\left(\left(U_{i}^{*}\right)^{n_{i}}-U_{i}\left(U_{i}^{*}\right)^{n_{i}+1}\right)=\prod_{i=1}^{d} \sum_{k_{i} \in \mathbb{N}}\left(\left(U_{i}^{*}\right)^{k_{i}}-U_{i}\left(U_{i}^{*}\right)^{k_{i}+1}\right)
$$

and using the fact that $\left(U_{1}, \ldots, U_{d}\right)$ is completely commuting.

\section{EXPANDING ENDOMORPHISMS OF THE $m$-DIMENSIONAL TORUS}

Let $A$ be a $m \times m(m \geq 1)$ matrix with integer entries. We say that $A$ is expanding if all its eigenvalues have modulus strictly greater than 1.

$A$ induces a transformation $\theta_{A}$ of the $m$-dimensional torus $[0,1)^{m}$, preserving the Lebesgue-Haar measure $\lambda$. We denote by $U_{A}$ the corresponding Koopman operator, and by $U_{A}^{*}$ the Perron-Frobenius operator.

Let us give a simple condition under which $\theta_{A}$ and $\theta_{B}$ are completely commuting.

Lemma 9. Let $A$ and $B$ be two expanding $m \times m(m \geq 1)$ matrices with integer entries. Assume that $A$ and $B$ commutes and that they have coprime determinants. Then, $\theta_{A}$ and $\theta_{B}$ are completely commuting. 
Proof. We have to prove that $U_{A}^{*} U_{B}=U_{B} U_{A}^{*}$. Let $\Gamma$ be representatives of $\mathbb{Z}^{m} / A \mathbb{Z}^{m}$ with distinct images in $\mathbb{Z}^{m} / A \mathbb{Z}^{m}$. By (34) (using that $A^{-1}$ and $B$ commute), it suffices to prove that $B$ induces a bijection (modulo $\mathbb{Z}^{m}$ ) of the set $\left(A^{-1} \gamma\right)_{\gamma \in \Gamma}$.

Let $\gamma, \gamma^{\prime} \in \Gamma$ be such that there exists $\beta \in \mathbb{Z}^{m}$ such that $B A^{-1} \gamma=B A^{-1} \gamma^{\prime}+\beta$. Set $\delta:=\gamma-\gamma^{\prime} \in \mathbb{Z}^{m}$. Writing $A^{-1}=(\operatorname{det} A)^{-1} \tilde{A}$, where $\tilde{A}$ is the adjugate matrix of $A$ (with integer entries) and similarly, $B^{-1}=(\operatorname{det} B)^{-1} \tilde{B}$, we see that

$$
(\operatorname{det} B) \tilde{A} \delta=(\operatorname{det} A) \tilde{B} \beta \text {. }
$$

Since $\operatorname{det} B \wedge \operatorname{det} A=1$, by Gauss lemma, we infer that $\operatorname{det} A \operatorname{divides}$ all entries $\tilde{A} \delta$, hence that $A^{-1} \delta \in \mathbb{Z}^{m}$ and $\delta \in A \mathbb{Z}^{m}$. By definition of $\Gamma$, we see that $\gamma=\gamma^{\prime}$ and the lemma is proved.

The fact that $A$ and $B$ have coprime determinants is by no mean necessary for $\theta_{A}$ and $\theta_{B}$ to be completely commuting as one may see from the following basic example: $A=\left(\begin{array}{ll}2 & 0 \\ 0 & 3\end{array}\right)$ and $B=\left(\begin{array}{ll}3 & 0 \\ 0 & 2\end{array}\right)$.

The next proposition is an easy consequence of a result by Fan [7] (see Proposition 13 of the Appendix). Recall that the modulus of continuity in $L^{2}$ is given by

$$
\Omega_{2, f}(\delta):=\sup _{0 \leq|x| \leq \delta}\|f(\cdot+x)-f\|_{2},
$$

where $|x|$ stands for the euclidean norm of $x \in[0,1)^{m}$.

Proposition 10. Let $A_{1}, \ldots, A_{d}$ be commuting expanding $m \times m$ matrices with integral entries. Let $\lambda_{\text {min }}>1$ be the infimum of the modulus of their eigenvalues. There exists $C>0$ such that, for every $f \in L^{2}(\lambda)$ and every $n_{1}, \ldots, n_{d} \in \mathbb{N}$,

$$
\left\|\left(U_{A_{1}}^{*}\right)^{n_{1}} \cdots\left(U_{A_{d}}^{*}\right)^{n_{d}} f\right\|_{2} \leq \Omega_{2, f}\left(C \lambda_{\min }^{-\left(n_{1}+\cdots+n_{d}\right)}\right) .
$$

Proof. Let us first notice that $\left(U_{A_{1}}^{*}\right)^{n_{1}} \cdots\left(U_{A_{d}}^{*}\right)^{n_{d}}$ is the Perron-Frobenius operator associated with $\theta_{A_{d}{ }^{n} \cdots A_{1} n_{1}}$. Now, since the matrices are commuting, it follows from standard linear algebra results, that the set of the eigenvalues of $A_{d}{ }^{n_{d}} \cdots A_{1}{ }^{n_{1}}$ is included in $\left\{\lambda_{d}{ }^{n_{d}} \cdots \lambda_{1}{ }^{n_{1}}: \lambda_{i}\right.$ is an eigenvalue of $\left.A_{i}\right\}$. In particular, $A_{d}{ }^{n_{d}} \cdots A_{1}{ }^{n_{1}}$ is an expanding matrix. Then, the result follows from Proposition 13, noticing that

$$
\Delta\left(A_{1}^{-n_{1}} \cdots A_{d}^{-n_{d}}\left([0,1]^{m}\right)\right) \leq C \lambda_{\min }^{-\left(n_{1}+\cdots+n_{d}\right)},
$$

where $\Delta\left(A_{1}^{-n_{1}} \cdots A_{d}^{-n_{d}}\left([0,1]^{m}\right)\right)$ is the diameter of $A_{1}^{-n_{1}} \cdots A_{d}^{-n_{d}}\left([0,1]^{m}\right)$.

We shall use the following notation: $U^{i}=U_{A_{1}}^{i_{1}} \cdots U_{A_{d}}^{i_{d}}$ for every $\left(i_{1}, \ldots, i_{d}\right) \in \mathbb{N}^{d}$.

Theorem 11. Let $d \geq 1$. Let $f \in L^{2}\left([0,1)^{m}, \lambda\right)$ centered such that

$$
\int_{0}^{1} \frac{|\log (t)|^{(d-2) / 2}}{t} \Omega_{2, f}(t) d t<\infty
$$

Let $A_{1}, \ldots, A_{d}$ be expanding $m \times m$ matrices with integral entries. Assume that they are commuting and that their determinants are pairwise coprime. Then, there exists 
a stationary $d$-field of reverse martingale differences $\left(U^{\underline{n}}(d)\right)_{\bar{n} \in \mathbb{N}^{d}}$ with $d \in L^{2}(\lambda)$ such that

$$
\mathbb{E}\left(\max _{1 \preceq \underline{k} \preceq \underline{n}}\left|\sum_{0 \preceq \underline{i} \preceq \underline{k}} U^{\underline{i}} f-U^{\underline{i}} d\right|^{2}\right)=o\left(n_{1} \cdots n_{d}\right) \quad \text { as } n_{1}, \ldots, n_{d} \rightarrow+\infty .
$$

In particular, we have an invariance principle.

Remark. We see that if $\Omega_{2, f}(t)=o\left(|\log (t)|^{-d / 2-\varepsilon}\right)(t \rightarrow 0)(25)$ holds. A CLT has been obtained by Cohen and Conze [2] under the condition $\Omega_{2, f}(t)=o\left(|\log (t)|^{-d-\varepsilon}\right)$ $(t \rightarrow 0)$. However, the results of [2] apply to general arrays and to commuting families of general endomorphisms (including for instance automorphisms) inducing a totally ergodic $\mathbb{N}^{d}$-action (see their paper for more informations).

Proof. It follows from (31) and a density argument that $\left\|\left(U_{A_{i}}^{*}\right)^{n} f\right\|_{2} \rightarrow 0$ as $n \rightarrow+\infty$ for every centered $f \in L^{2}(\lambda)$ and every $i \in\{1, \ldots, d\}$. Hence, by Theorem 8 , we just have to check that (14) holds. Using (31), we see that (14) will hold provided that

$$
\sum_{n_{1}, \ldots, n_{d} \in \mathbb{N}^{*}} \frac{\Omega_{2, f}\left(C \lambda_{\min }^{-\left(n_{1}+\cdots+n_{d}\right)}\right)}{\left(n_{1} \cdots n_{d}\right)^{1 / 2}}<\infty .
$$

Now, making the change of index $n_{1}+\cdots+n_{d} \rightarrow n_{d}$,

$$
\begin{aligned}
\sum_{n_{1}, \ldots, n_{d} \in \mathbb{N}^{*}} \frac{\Omega_{2, f}\left(C \lambda_{\min }^{-\left(n_{1}+\cdots+n_{d}\right)}\right)}{\left(n_{1} \cdots n_{d}\right)^{1 / 2}} & \\
& =\sum_{n_{1}, \ldots, n_{d-1} \in \mathbb{N}^{*}} \sum_{n_{d} \geq n-1+\cdots+n_{d-1}+1} \frac{\Omega_{2, f}\left(C \lambda_{\min }^{-n_{d}}\right)}{\left(n_{1} \cdots\left(n_{d}-n_{1}-\cdots-n_{d-1}\right)\right)^{1 / 2}} .
\end{aligned}
$$

Notice that for every $m \geq 1$, we have

$$
\sum_{1 \leq n_{1} \leq m-1} \frac{1}{\sqrt{n_{1}\left(m-n_{1}\right)}} \leq \sqrt{2 / m} \sum_{1 \leq n_{1} \leq m / 2} \frac{1}{\sqrt{n_{1}}}+\sqrt{2 / m} \sum_{m / 2 \leq n_{1} \leq m-1} \frac{1}{\sqrt{m-n_{1}}} \leq D,
$$

for a constant $D>0$ independent of $m$.

Hence (27) holds if and only if

$$
\begin{gathered}
\sum_{n_{d} \geq 1} \sum_{n_{2}, \ldots, n_{d-1} \in \mathbb{N}^{*}: n_{2}+\cdots+n_{d-1} \leq n_{d}-1} \frac{\Omega_{2, f}\left(C \lambda_{\text {min }}^{n_{d}}\right)}{\sqrt{n_{2} \cdots n_{d-2}}} \\
\leq \sum_{n_{d} \geq 1}\left(\sum_{1 \leq j \leq n_{d}} \frac{1}{\sqrt{j}}\right)^{d-2} \Omega_{2, f}\left(C \lambda_{\text {min }}^{n_{d}}\right) \leq C \sum_{n_{d} \geq 1} n_{d}^{(d-2) / 2} \Omega_{2, f}\left(C \lambda_{\text {min }}^{n_{d}}\right)<\infty,
\end{gathered}
$$

which is equivalent to (25) by comparing series and integrals.

We shall now give a sufficient condition in terms of Fourier coefficients. 
Lemma 12. Let $f \in L^{2}\left([0,1)^{m}\right)$ with Fourier coefficients $\left(c_{\underline{n}}(f)\right)_{\underline{n} \in \mathbb{Z}^{m}}$. For every $k \geq 1$, define

$$
A_{k}(f):=\max _{1 \leq i \leq m} \sum_{\underline{n} \in \mathbb{Z}^{m}:\left|n_{i}\right| \geq k}\left|c_{n}(f)\right|^{2} .
$$

If

$$
\sum_{k \geq 1} \frac{(\log k)^{(d-2) / 2}}{k}\left(A_{k}(f)\right)^{1 / 2}<\infty
$$

then (25) holds.

Remark. Note that (28) holds as soon as $A_{k}(f)=O\left((\log k)^{-d-\varepsilon}\right)$, for some $\varepsilon>0$. Conversely, if (28) is satisfied, since $\left(A_{k}(f)\right)_{k \geq 1}$ is non increasing, we see that, $A_{k}(f)=$ $o\left((\log k)^{-d}\right)$. Note also that Levin [16] proved the CLT (he also announced the weak invariance principle in [16]) under a condition that is easily seen to be equivalent to

$$
\sum_{k \geq 1} \frac{(\log k)^{d-1}}{k}\left(A_{k}(f)\right)^{1 / 2}<\infty .
$$

He worked in the same setting as Cohen and Conze [2].

Proof. Let us first notice that (25) is equivalent to

$$
\sum_{k \geq 1} \frac{(\log k)^{(d-2) / 2}}{k} \Omega_{2, f}(1 / k)<\infty .
$$

Let $k \geq 1$ and $\underline{x} \in \mathbb{R}^{m}$ with $|\underline{x}| \leq 1 / k$. We have

$$
\|f(\cdot+\underline{x})-f\|_{2}^{2}=\sum_{\underline{n} \in \mathbb{Z}^{m}}\left|c_{\underline{n}}(f)\right|^{2}\left|1-\mathrm{e}^{2 i \pi\langle\underline{n}, \underline{x}\rangle}\right|^{2} .
$$

In particular, majorizing $\left|1-\mathrm{e}^{2 i \pi\langle\underline{n}, \underline{x}\rangle}\right|$ either by 2 or by $2 \pi|\underline{n}||\underline{x}|$, we see that

$$
\begin{aligned}
\left(\Omega_{2, f}(1 / k)\right)^{2} \leq & \sum_{\underline{n} \in\{-k, \ldots, k\}^{m}}\left|c_{\underline{n}}(f)\right|^{2} \frac{4 \pi^{2}|\underline{n}|^{2}}{k^{2}}+C_{m} \sum_{i=1}^{m} \sum_{\underline{n} \in \mathbb{Z}^{m}:\left|n_{i}\right| \geq k}\left|c_{\underline{n}}(f)\right|^{2} \\
& \leq \frac{\tilde{C}_{m}}{k^{2}} \sum_{i=1}^{m} \sum_{\underline{n} \in \mathbb{Z}^{m}:\left|n_{i}\right| \leq k} n_{i}^{2}\left|c_{\underline{n}}(f)\right|^{2}+C_{m} \sum_{i=1}^{m} \sum_{\underline{n} \in \mathbb{Z}^{m}:\left|n_{i}\right| \geq k}\left|c_{\underline{n}}(f)\right|^{2},
\end{aligned}
$$

for two positive constants $C_{m}$ and $\bar{C}_{m}$. The second sum on right hand can be handled directly by using (28), and it remains to prove that

$$
\sum_{k \geq 1} \frac{(\log k)^{(d-2) / 2}}{k^{2}}\left(B_{k}(f)\right)^{1 / 2}<\infty
$$

where

$$
B_{k}(f):=\max _{1 \leq i \leq m} \sum_{\underline{n} \in \mathbb{Z}^{m}:\left|n_{i}\right| \leq k} n_{i}^{2}\left|c_{n}(f)\right|^{2} .
$$


Let us prove that (28) implies (29). Let

$$
A_{k}^{(i)}:=\sum_{\underline{n} \in \mathbb{Z}^{m}:\left|n_{i}\right| \geq k}\left|c_{n}(f)\right|^{2} .
$$

We have

$$
\sum_{\underline{n} \in \mathbb{Z}^{m}:\left|n_{i}\right| \leq k} n_{i}^{2}\left|c_{n}(f)\right|^{2}=\sum_{j=0}^{k} j^{2}\left(A_{j}^{(i)}-A_{j+1}^{(i)}\right)=\sum_{j=1}^{k} A_{j}^{(i)}\left(j^{2}-(j-1)^{2}\right)-k^{2} A_{k+1}^{(i)} .
$$

Hence, we infer that (29) hold as soon as

$$
B:=\sum_{k \geq 1} \frac{(\log k)^{(d-2) / 2}}{k^{2}}\left(\sum_{j=1}^{k} j A_{j}(f)\right)^{1 / 2}<\infty
$$

To prove that (28) implies (30), we first notice that (28) is equivalent to

$$
\sum_{n \geq 0} n^{(d-2) / 2} \sqrt{A_{2^{n}}(f)}<\infty
$$

(to see this it suffices to use the monoticity of $A_{k}$ ). Next

$$
B=\sum_{n=0}^{\infty} \sum_{k=2^{n}}^{2^{n+1}} \frac{(\log k)^{(d-2) / 2}}{k^{2}}\left(\sum_{j=1}^{k} j A_{j}(f)\right)^{1 / 2} \leq C \sum_{n=0}^{\infty} \frac{n^{(d-2) / 2}}{2^{n}}\left(\sum_{j=1}^{2^{n+1}} j A_{j}(f)\right)^{1 / 2} .
$$

In the same way, using the monoticity of $A_{k}$,

$$
\left(\sum_{j=1}^{2^{n+1}} j A_{j}(f)\right)^{1 / 2}=\left(\sum_{k=0}^{n} \sum_{j=2^{k}}^{2^{k+1}} j A_{j}\right)^{1 / 2} \leq\left(2 \sum_{k=0}^{n} 2^{2 k} A_{2^{k}}\right)^{1 / 2} \leq \sqrt{2} \sum_{k=0}^{n} 2^{k} \sqrt{A_{2^{k}}} .
$$

It follows that

$$
B \leq C \sum_{n=0}^{\infty} \frac{n^{(d-2) / 2}}{2^{n}} \sum_{k=0}^{n} 2^{k} \sqrt{A_{2^{k}}} \leq D \sum_{k \geq 0} k^{(d-2) / 2} \sqrt{A_{2^{k}}(f)}<\infty
$$

which completes the proof.

\section{Appendix}

Proposition 10 of Section 5 is a consequence of the following proposition, due to Fan [7]. We shall give the proof for the sake of completeness and because the reference [7] is hard to obtain.

Proposition 13 (Fan, [7]). There exists $C>0$ such that for every expanding $m \times m$ matrices $A$ with integral entries, for every $f \in L^{2}(\lambda)$ with $\lambda(f)=0$,

$$
\left\|U_{A}^{*} f\right\|_{2} \leq \Omega_{2, f}\left(\Delta\left(A^{-1}\left([0,1]^{m}\right)\right)\right),
$$

where $\Delta\left(A^{-1}\left([0,1]^{m}\right)\right)$ is the diameter of $A^{-1}\left([0,1]^{m}\right)$. 
Proof. Let us recall some facts about tiling and Perron-Frobenius operators associated with expanding matrices.

Let $A$ be an expanding $m \times m$ matrix. Let $\Gamma \subset \mathbb{Z}^{m}$ be representatives of $\mathbb{Z}^{m} / A \mathbb{Z}^{m}$. Then, see e.g. [3], there exists a unique compact set $K \subset \mathbb{R}^{m}$, such that

$$
K=\cup_{\gamma \in \Gamma}\left(A^{-1} K+A^{-1} \gamma\right)
$$

and an integer $q \geq 1$ such that

$$
\sum_{\underline{n} \in \mathbb{Z}^{m}} \mathbf{1}_{K+\underline{n}}=q \quad \lambda \text {-almost everywhere. }
$$

Moreover, for every $\gamma, \gamma^{\prime} \in \Gamma$ with $\gamma \neq \gamma^{\prime}, \lambda\left(\left(A^{-1} K+A^{-1} \gamma\right) \cap\left(A^{-1} K+A^{-1} \gamma^{\prime}\right)\right)=0$. Using that, clearly,

$$
\mathbf{1}_{K}=\sum_{\underline{n} \in \mathbb{Z}^{d}} \mathbf{1}_{\left((K+\underline{n}) \cap[0,1]^{m}\right)-\underline{n}},
$$

we infer that for every locally integrable $f$,

$$
q \int_{[0,1]^{m}} f d \lambda=\int_{K} f d \lambda
$$

It follows then that the Perron-Frobenius operator $U_{A}^{*}$ is given by

$$
U_{A}^{*} f(x)=\frac{1}{\operatorname{det} A} \sum_{\gamma \in \Gamma} f\left(A^{-1} x+A^{-1} \gamma\right) .
$$

Let $f \in L^{2}(\lambda)$ with $\lambda(f)=0$. Using that $U_{A}^{*}$ preserves $\lambda$ and making the change of variable $y \rightarrow y+x$, we infer that

$$
U_{A}^{*} f(x)=\frac{1}{q \operatorname{det} A} \sum_{\gamma \in \Gamma} \int_{K}\left(f\left(A^{-1} x+A^{-1} \gamma\right)-f\left(A^{-1}(y+x)+A^{-1} \gamma\right)\right) \lambda(d y) .
$$

Hence, using Jensen's inequality (recall that $\lambda(K)=q$ ),

$$
\left(U_{A}^{*} f\right)^{2} \leq \frac{1}{q \operatorname{det} A} \sum_{\gamma \in \Gamma} \int_{K}\left(f\left(A^{-1} x+A^{-1} \gamma\right)-f\left(A^{-1}(y+x)+A^{-1} \gamma\right)\right)^{2} \lambda(d y) .
$$

Making the change of variable $x \rightarrow A^{-1} x+A^{-1} \gamma$, we infer that

$$
\begin{aligned}
\left\|U_{A}^{*} f\right\|_{2}^{2} \leq \sum_{\gamma \in \Gamma}\left(\int_{K} \lambda(d y) \int_{K}\left(f\left(A^{-1} x+A^{-1} \gamma\right)-f\left(A^{-1}(y+x)+A^{-1} \gamma\right)\right)^{2} \lambda(d x)\right)^{1 / 2} \\
\leq \frac{1}{q^{2}} \sum_{\gamma \in \Gamma} \int_{K} \lambda(d y) \int_{A^{-1} K+A^{-1} \gamma}\left(f(x)-f\left(x+A^{-1} y\right)\right)^{2} \lambda(d x) \\
=\int_{[0,1]^{m}}\left\|f-f\left(\cdot+A^{-1} y\right)\right\|_{2}^{2} \lambda(d y),
\end{aligned}
$$

where we have used (32) and (33). The announced result clearly follows. 


\section{REFERENCES}

[1] R. Cairoli, Une inégalité pour martingales à indices multiples et ses applications (French) Séminaire de Probabilités, IV (Univ. Strasbourg, 1968/69) (1970) 1-27 Lecture Notes in Mathematics, Vol. 124. Springer, Berlin.

[2] G. Cohen and J.-P. Conze, CLT for random walks of commuting endomorphisms on compact abelian groups, accepted for publicaion in J. Theoret. Probab, DOI: 10.1007/s10959-015-0631-y.

[3] J.-P. Conze, L. Hervé, L. and A. Raugi, Pavages auto-affines, opérateurs de transfert et critères de réseau dans $\mathbb{R}^{d}$, (French) Bol. Soc. Brasil. Mat. (N.S.) 28 (1997), no. 1, 1-42.

[4] C. Cuny, A compact LIL for martingales in 2-smooth Banach spaces with applications, Bernoulli 21 (2015), no. 1, 374-400.

[5] C. Cuny and M. Peligrad, Central limit theorem started at a point for stationary processes and additive functionals of reversible Markov chains, J. Theoret. Probab. 25 (2012), no. 1, 171-188.

[6] J. Dedecker, On the optimality of McLeish's conditions for the central limit theorem, C. R. Math. Acad. Sci. Paris 353 (2015), no. 6, 557-561.

[7] A. Fan, Decay of correlation for expanding toral endomorphisms.

[8] M. I. Gordin, The central limit theorem for stationary processes, (Russian) Dokl. Akad. Nauk SSSR 1881969 739-741.

[9] M. I. Gordin, Martingale-co-boundary representation for a class of stationary random fields, (Russian) Zap. Nauchn. Sem. S.-Peterburg. Otdel. Mat. Inst. Steklov. (POMI) 364 (2009), Veroyatnost i Statistika. 14.2, 88-108, 236; translation in J. Math. Sci. (N. Y.) 163 (2009), no. 4, 363-374.

[10] M. I. Gordin and B. A. Lifšic, Central limit theorem for stationary Markov processes, (Russian) Dokl. Akad. Nauk SSSR 239 (1978), no. 4, 766-767.

[11] M. I. Gordin and M. Peligrad, On the functional central limit theorem via martingale approximation, Bernoulli 17 (2011) 424-440.

[12] P. Hall and C. C. Heyde, Martingale limit theory and its application. Probability and Mathematical Statistics, Academic Press, Inc. [Harcourt Brace Jovanovich, Publishers], New York-London, 1980.

[13] E. J. Hannan, Central limit theorems for time series regression, Z. Wahrscheinlichkeitstheorie und Verw. Gebiete 26 (1973) 157-170.

[14] E. J. Hannan, The central limit theorem for time series regression, Stochastic Process. Appl. 9 (1979) 281-289.

[15] C. C. Heyde, On the central limit theorem for stationary processes, Z. Wahrscheinlichkietstheorie und Verw. Gebiete 30 (1974), 315-320.

[16] M. B. Levin, Central limit theorem for Zd+-actions by toral endomorphisms, Electron. J. Probab. 18 (2013), no. 35, 42 pp.

[17] M. Maxwell and M. Woodroofe, Central limit theorems for additive functionals of Markov chains, Ann. Probab. 28 (2000) 713-724.

[18] M. Peligrad and S. Utev, A new maximal inequality and invariance principle for stationary sequences, Ann. Probab. 33 (2005) 798-815.

[19] D. Volný, A central limit theorem for fields of martingale differences, arXiv:1504.02439, accepted for publication in C. R. Math. Acad. Sci. Paris

[20] D. Volný and Y. Wang, An invariance principle for stationary random fields under Hannan's condition, Stochastic Process. Appl. 124 (2014), no. 12, 4012-4029.

[21] P. Walters, An introduction to ergodic theory. Graduate Texts in Mathematics, 79. SpringerVerlag, New York-Berlin, 1982. 
Laboratoire Mas, Centrale-Supelec, Grande Voie des Vignes, 92295 Chatenay-Malabry CEDEX, FRANCE

E-mail address: christophe.cuny@ecp.fr

Laboratoire MAP5 (UMR 8145), Université Paris Descartes, Sorbonne Paris Cité, 45 Rue des SAInts PÈres, 75270 PARIS CEDEx 06, FRANCE

E-mail address: jerome.dedecker@parisdescartes.fr

Laboratoire de Mathématiques Raphä̈l Salem (UMR 6085), Université de Rouen, Avenue de L'Universit, BP.12 76801 Saint-Etienne DU Rouvray, FRANCE

E-mail address: dalibor.volny@univ-rouen.fr 